\title{
WILEY-VCH
}

DOI: $10.1002 /(($ please add manuscript number $))$

Article type: Communication

\section{A living-dead magnetic layer at the surface of ferrimagnetic $\mathrm{DyTiO}_{3}$ thin films}

Raphaël Aeschlimann, Daniele Preziosi ${ }^{\star}$, Philipp Scheiderer, Michael Sing, Sergio Valencia, Jacobo Santamaria, Chen Luo, Hanjo Ryll, Florin Radu, Ralph Claessen, Cinthia Piamonteze and Manuel Bibes*

Raphaël Aeschlimann, Dr. Daniele Preziosi and Dr. Manuel Bibes

Unité Mixte de Physique, CNRS, Thales, Université Paris-Saclay, 91767 Palaiseau, FRANCE

E-mail: manuel.bibes@cnrs-thales.fr

Philipp Scheiderer, Prof. Michael Sing, Prof. Ralph Claessen

Physikalisches Institut and Röntgen Center for Complex Material Systems (RCCM), Universität Würzburg, Am Hubland, D-97074 Würzburg, GERMANY

Dr. Sergio Valencia, Dr. Chen Luo, Dr. Hanjo Ryll, Dr. Florin Radu

Helmholtz-Zentrum Berlin für Materialen \& Energie, Albert-Einstein-Strasse 15, 12489

Berlin, GERMANY

Prof. Jacobo Santamaria

GFMC, Dpto. Física de Materiales, Universidad Complutense de Madrid, 28040, SPAIN

Dr. Chen Luo

Institut für Experimentelle und Angewandte Physik, Universität Regensburg,

Universitätsstrasse 31, D-93053 Regensburg, GERMANY

Dr. Cinthia Piamonteze

Swiss Light Source, Paul Scherrer Institut, CH-5232 Villigen PSI, SWITZERLAND

- Now at Institut de Physique et Chimie des Matériaux de Strasbourg, Université de Strasbourg, CNRS, UMR 7504, 67000 Strasbourg, France.

Keywords: magnetic oxides, Mott insulators, X-ray absorption spectroscopy, X-ray photoemission spectroscopy 


\section{WILEY-VCH}

\section{Abstract}

When ferromagnetic films become ultrathin, key properties such as the Curie temperature and the saturation magnetization are usually depressed. This effect has been thoroughly investigated in magnetic oxides such as half-metallic manganites, but much less in ferrimagnetic insulating perovskites such as rare-earth titanates $\mathrm{RTiO}_{3}$, despite the appeal of these materials to design correlated two-dimensional electron gases. Here, we report on the magnetic properties of epitaxial $\mathrm{DyTiO}_{3}$ thin films. While films thicker than about $50 \mathrm{~nm}$ show a bulk-like response, at low thickness we observe a surprising increase of the saturation magnetization. We model this behavior using a classical model of "dead layer" but we assume that this layer is actually "living", i.e. it responds to the magnetic field with a strong paramagnetic susceptibility. Through depth-dependent X-ray absorption and photoemission spectroscopy, we show that the "living-dead layer" corresponds to surface regions where magnetic $(\mathrm{S}=1 / 2) \mathrm{Ti}^{3+}$ ions are replaced by non-magnetic $\mathrm{Ti}^{4+}$ ions. Element specific hysteresis cycles obtained at the $\mathrm{Dy} \mathrm{M}_{5}$ and $\mathrm{Ti} L_{3}$ edges indicate that the surface $\mathrm{Ti}^{4+}$ ions decouple the $\mathrm{Dy}^{3+}$ ions, thus unleashing their strong paramagnetic response. Finally, we show how capping the $\mathrm{DyTiO}_{3}$ film with different materials can help increase the $\mathrm{Ti}^{3+}$ content near the surface and thus recover a better ferrimagnetic behavior. 


\section{WILEY-VCH}

Among transition-metal perovskites, rare-earth orthorhombic titanates $\mathrm{RTiO}_{3},(\mathrm{R}=\mathrm{La} \ldots$... Lu or Y) emerge as a particularly interesting family of Mott insulators ${ }^{[1-4]}$. The most stable valence state of rare-earths (except for $\mathrm{Eu}$ and $\mathrm{Ce}$ ) is 3+, which imposes a 3+ valence state to the $\mathrm{Ti}$, that is thus in a spin $1 / 23 \mathrm{~d}^{1}$ electronic state. A salient feature of $\mathrm{RTiO}_{3}$ compounds is the phase transition from ferrimagnetic to antiferromagnetic (G-type) order when going from small (Lu... Gd) to large rare-earths $(\mathrm{Sm} . . \mathrm{La})^{[1,3]}$. This insulating-ferrimagnetic behavior is exceptional among simple perovskites and offers perspectives for engineering novel magnetic states at oxide interfaces $^{[5,6]}$.

However, the growth of high quality thin films of rare-earth titanates is challenging due to the relatively poor stability of the $\mathrm{Ti} 3+$ valence state $^{[7]}$. So far, literature on ferrimagnetic titanate thin films is very scarce, aside from a few reports on $\mathrm{GdTiO}_{3}{ }^{[8,9]}$ and $\mathrm{YTiO}_{3}{ }^{[10,11]}$. Here, we focus on $\mathrm{DyTiO}_{3}$ (DTO), for which thin film growth has never been reported. In the bulk, this compound has a Curie temperature $\left(\mathrm{T}_{\mathrm{C}}\right)$ of $60 \mathrm{~K}$, among the highest in the $\mathrm{RTiO}_{3}$ family, and a non-collinear magnetic order comprising canted Dy moments whose resulting magnetization is antiferromagnetically coupled to that of $\mathrm{Ti}$ spins $^{[12,13]}$, leading to a saturation moment $\mathrm{M}_{\mathrm{S}}=3.7$ $\mu_{\mathrm{B}} /$ f.u. (f.u.: formula unit) ${ }^{[1]}$. Unlike in the better studied $\mathrm{GdTiO}_{3}$, the magnetic hysteresis cycles of DTO are very square with a strong remanence and a high coercive field ${ }^{[1]}$, making it more appealing to interfacially imprint a clear magnetic response onto an adjacent oxide layer ${ }^{[6]}$ for instance.

We report on the epitaxial deposition of single-phase epitaxial thin films of DTO using pulsed laser deposition (PLD) in two different growth conditions both corresponding to very low oxygen partial pressures $\left(\mathrm{P}_{\mathrm{O} 2}\right)$. We show that while thick films $(>50 \mathrm{~nm})$ have almost bulk-like magnetic properties, with a high remanence and a Curie temperature near $60 \mathrm{~K}$, in thinner films a paramagnetic component develops at the expense of the ferromagnetic one. By combining in situ X-ray photoelectron spectroscopy (XPS) and ex situ X-ray absorption spectroscopy (XAS) using several detection modes we could gain insight on the depth dependence of the Ti valence. 


\section{WILEY-VCH}

Through the correlation between the thickness dependence of the magnetic properties and the depth dependence of Ti oxidation state, we infer how overoxidized Ti ions at the film surface alter the coupling between Dy ions, yielding a peculiar increase of the high-field magnetization in ultrathin DTO films. We present X-ray reflectometry magnetic hysteresis cycles at the Dy $\mathrm{M}_{5}$ and $\mathrm{Ti} \mathrm{L}_{3}$ edges that confirm this picture. Finally, we explore the effect of epitaxial capping layers on the $\mathrm{Ti}$ valence and the magnetic properties.

Epitaxial thin films of DTO $(110)_{\mathrm{o}}$ (o : orthorhombic notation) have been grown on $\mathrm{LaAlO}_{3}$ $(001)_{\mathrm{pc}}$ (pc : pseudo-cubic notation) substrates by means of pulsed laser deposition (PLD) in a chamber with a background pressure was in the low $10^{-89}$ mbar range at room temperature and using a multiphase ceramic target ablated by a $\mathrm{KrF}$ excimer laser at $2 \mathrm{~Hz}$. The growth of rareearth titanate thin films requires a low $\mathrm{P}_{\mathrm{O} 2}$ to stabilize the $3+$ oxidation of $\mathrm{Ti}$ and avoid oxygen interstitials leading to a $\mathrm{Ti}^{4+}$ state $^{[7-9]}$. We found two suitable growth atmospheres : one under a total pressure of pure oxygen of $4 \times 10^{-7}$ mbar and one under an argon atmosphere of $1 \times 10^{-3}$ mbar, both with a substrate temperature of $900^{\circ} \mathrm{C}$. The growth was monitored in situ by reflection high-energy electron diffraction (RHEED). For both growth conditions, we observe oscillations of the specular beam intensity (cf insets of Figure 1a and b), indicative of a layerby-layer growth, slightly clearer for growth in pure Ar.

Figure 1a and 1b shows X-ray diffraction (XRD) $2 \theta-\omega$ scans for DTO films with a thickness ranging from 5 to $80 \mathrm{~nm}$ grown in pure $\mathrm{Ar}$ and low $\mathrm{P}_{\mathrm{O} 2}$, respectively. In addition to the LAO substrate $(001)_{\mathrm{pc}}$ reflections, we only observe peaks corresponding to the DTO $(110)_{\mathrm{pc}}$ reflections. The out-of-plane lattice constant is between $3.907 \pm 0.009 \AA$ for all films. For both sets of growth conditions, we thus managed to obtain single phase films of DTO over the full range of thicknesses.

We investigated the magnetic properties of those films through SQUID magnetometry (SQUID: superconducting quantum interference device) after field cooling in 100 Oe. Figure 1c and 1d display the field dependence of the magnetization at $10 \mathrm{~K}$ for films grown in pure Ar and in 


\section{WILEY-VCH}

low oxygen pressure, respectively. Figure 1e and $\mathbf{1 f}$ show the temperature dependence of the magnetization at 100 Oe. Thicker films show a clear hysteresis with a saturation moment at 50 kOe $\mathrm{M}_{\mathrm{S}}=3.5-3.7 \mu_{\mathrm{B}} /$ f.u., a large remanence and a ferromagnetic transition near $60 \mathrm{~K}$, consistent with the bulk $\mathrm{M}_{\mathrm{S}}$ and $\mathrm{T}_{\mathrm{C}}{ }^{[1]}$. As thickness decreases, $\mathrm{T}_{\mathrm{C}}$ is gradually reduced for films grown in oxygen whereas it stays more or less constant down to $5 \mathrm{~nm}$ for the films grown in pure Ar. In parallel, thickness reduction promotes a puzzling change in the $\mathrm{M}(\mathrm{H})$ loops: the loops become less square and more slanted with both remanence and coercivity decreasing, but the high-field magnetization increases up to more than $6 \mu_{\mathrm{B}} / \mathrm{f}$.u. This behavior is at odds with the situation in ferromagnetic manganites for instance, in which thickness reduction yields a concomitant decrease of both $T_{C}$ and $M_{S}$, the thinnest films showing no magnetic response ${ }^{[14,15]}$. These results are summarized in Figure 2. While for both sample series the remanent magnetization monotonically decreases as thickness is reduced (Figures $2 \mathrm{a}$ and 2c), the saturation magnetization shows an opposite behavior and tends to increase at low thickness (Figures $2 \mathrm{~b}$ and $2 \mathrm{~d})$.

In classical itinerant ferromagnets or double-exchange systems such as manganites, the reduction of the saturation magnetization in ultrathin films has been modelled using the socalled "dead-layer" picture that assumes that a fraction of the film - near the interface with the substrate or at the film surface - is non-magnetic (i.e. its magnetization is zero). In $\mathrm{La}_{2 / 3} \mathrm{Sr}_{1 / 3} \mathrm{MnO}_{3}$ the dead layer at the interface with an insulating perovskite such as $\mathrm{SrTiO}_{3}$ has a typical thickness of a few $\mathrm{nm}^{[14,16,17]}$. It is believed to originate from changes in the optimal carrier density (due to charge transfer from the adjacent material and/or to the presence of oxygen vacancies) that drive the material's ground state towards neighboring antiferromagnetic and insulating states of the (bulk) phase diagram ${ }^{[16-18]}$.

While our experimental results also suggest that when thickness is reduced the relative contribution of the ferrimagnetic phase of DTO to the overall magnetic response decreases at the expense of another one, they are clearly incompatible with a picture assuming such a non- 


\section{WILEY-VCH}

magnetic dead layer. Instead, we propose that the observed behavior arises from the existence of a non-ferromagnetic "living-dead layer", responding to the field with a strong paramagnetic susceptibility, as suggested by the shape of the $\mathrm{M}(\mathrm{H})$ loops in Figure 1c and 1d.

To substantiate our claim, we fit the thickness dependence of the remanent and saturation magnetization with this living-dead layer model. Although some dead layer may also be present at the substrate/film interface, we hypothesize that the response is dominated by a dead layer at the film surface. We assume that this layer is paramagnetic and thus has a null magnetization at remanence and a large magnetization at high field. We use a simple bilayer model (a ferrimagnetic layer and a paramagnetic layer):

$$
\begin{gathered}
M_{\text {total }}=m_{\text {total }} \cdot t=m_{P} \cdot t_{P}+m_{F} \cdot t_{F} \\
m_{\text {total }}=\frac{\left(m_{P}-m_{F}\right) \cdot t_{P}}{t}+m_{F}
\end{gathered}
$$

with $t=t_{F}+t_{P}$ the total film thickness, $t_{F}$ the thickness of the ferrimagnetic layer, $t_{P}$ the thickness of the paramagnetic layer, $m_{F}$ the moment per unit volume of the ferrimagnetic layer and $m_{P}$ the moment per unit volume of the paramagnetic layer. We used the DTO bulk value $3.7 \mu_{\mathrm{B}} / \mathrm{f}_{\mathrm{u}} \mathrm{u}^{[1]}$ for $m_{F}$ and focus on the samples grown in pure Ar. Setting $m_{P}$ to the maximum magnetization of the thinnest sample $\left(m_{P} \approx 6.5 \mu_{\mathrm{B}}\right)$ and using $t_{p}$ as a fitting parameter (model 1) yields a high fit quality, with $t_{p}=7-8 \mathrm{~nm}$ (Fig. 2a). A dead layer thickness of $5 \mathrm{~nm}$ is obtained by fitting the remanent magnetization (Fig. 2a) and assuming here $m_{P}=0 \mu_{\mathrm{B}}$. For the $\mathrm{O}_{2}$ grown films, the fit of $M_{R}$ (not shown) is not very good, possibly reflecting a progressive reduction of the anisotropy in the ferrimagnetic layer when decreasing thickness. We will come back to these data later on. We now turn to the origin of this dead layer. To address this issue, we have investigated the valence of the atomic species present in the film using X-ray photoelectron spectroscopy (XPS) and X-ray absorption spectroscopy (XAS) with three detection modes probing the sample over different depths. In Figure 3 we plot the XPS spectra of Ti 2p core levels for two $20 \mathrm{~nm}$ films collected at different angles, allowing to vary the probing depth over about $5 \mathrm{~nm}$. Focusing on 


\section{WILEY-VCH}

the spectra for the pure Ar film (Fig. 3a and 3b), we notice that each of the Ti $2 \mathrm{p}_{1 / 2}$ and $2 \mathrm{p}_{3 / 2}$ peaks comprises two main components: one corresponding to $\mathrm{Ti}^{4+}$ (shaded in blue) and another to $\mathrm{Ti}^{3+}$ (shaded in red) at higher and lower binding energies, respectively. A third, minor component, possibly corresponding to $\mathrm{Ti}^{2+}$ (in green) is also visible ${ }^{[19,20]}$. At normal emission (Fig. 3a), the $3+/ 4+$ ratio is $80: 20$ and decreases to $39: 61$ at $45^{\circ}$ emission. The data thus clearly point to a gradient of $\mathrm{Ti}$ valence over the sample depth, with a higher concentration of $\mathrm{Ti}^{4+}$ near the surface ${ }^{[21]}$. Following Ref. ${ }^{[22]}$, we estimate that the equivalent thickness of the $\mathrm{Ti}^{4+}$ surface layer is about $1.3 \mathrm{~nm}$. For the film grown in $\mathrm{O}_{2}$, the XPS spectrum at normal emission (Fig. 3c) suggests a larger proportion of $\mathrm{Ti}^{4+}(3+/ 4+$ ratio is $\sim 20: 80)$, in line with the more degraded magnetic response of films grown in $\mathrm{O}_{2}$ in this thickness range.

Further insight into this depth dependence of the Ti valence state is provided by XAS on a 80 $\mathrm{nm}$ film. We have acquired spectra using three different detection modes: total electron yield (TEY, probing depth $\sim 2-10 \mathrm{~nm}$ ), total fluorescence yield (TFY, probing depth a few tens of nm) and X-ray excited optical luminescence (XEOL, the whole film thickness is probed). In TEY (Fig. 3d), the spectrum exhibits four main features corresponding to the transition from $2 \mathrm{p}_{3 / 2}\left(L_{3}\right.$ edge $)$ and $2 \mathrm{p}_{1 / 2}\left(L_{2}\right.$ edge $)$ levels to the $3 \mathrm{~d}$ levels split into $\mathrm{t}_{2 \mathrm{~g}}$ and $\mathrm{e}_{\mathrm{g}}$ states by the crystal field. The spectrum looks close to the one expected from $\mathrm{Ti}^{4+}$ (as in $\mathrm{SrTiO}_{3}$ for example ${ }^{[23]}$ ), with albeit some noticeable differences in line shape and intensities. As we probe deeper in the sample using TFY (Fig. 3e), the spectrum exhibits additional components, and peaks at lower energy grow in intensity ${ }^{[24,25]}$. We interpret this as the signature of $\mathrm{Ti}^{3+}$ ions for which XAS features shift to lower photon energies due to the smaller binding to the $2 \mathrm{p}$ levels and present a richer multiplet structure due to the splitting of the $t_{2 g}$ and $e_{g}$ states, expected for a $3 d^{1}$ configuration. Finally, the XEOL spectrum (Fig. 3f) suggest the presence of an almost pure 3+ state $^{[21,26]}$, very rarely reported in the literature (presumably because most XAS experiments used TEY detection and thus only probed the $\mathrm{Ti}^{4+}$ rich dead layer at the surface ${ }^{[27]}$ ). 


\section{WILEY-VCH}

The data in Fig. 3d-f provide information ${ }^{[28]}$ on the effective volume fraction of regions in which $\mathrm{Ti}$ is $4+$. Fig. $3 \mathrm{~g}$ presents linear combinations of pure $\mathrm{Ti}^{4+[23]}$ and "pure" $\mathrm{Ti}^{3+} \mathrm{XAS}$ spectra (we use our XEOL data for which Ti is very close to pure 3+). By comparison with the experimental spectra we can estimate that the XAS data collected in TEY correspond approximately to a 80:20 $\mathrm{Ti}^{4+}: \mathrm{Ti}^{3+}$ ratio and in FY to a 10:90 ratio. Since in TEY the probing depth is about $5 \mathrm{~nm}$, this indicates that a region near the film surface with an effective thickness of $\sim 4 \mathrm{~nm}$ contains $\mathrm{Ti}^{4+}$ only. This layer contributes to the XAS in TFY, and the 10:90 ratio suggests a probing depth of $\sim 40 \mathrm{~nm}$ in TFY, which is reasonable. The good correspondence between this equivalent thickness of pure $\mathrm{Ti}^{4+}$ and the thickness of the magnetic living-dead layer strongly suggests that the deviation from a $3+$ valence near the film surface triggers the paramagnetic response of the Dy ions.

To test our hypothesis of a link between the titanium valence state and the observed paramagnetic signal, we measured the magnetic field dependence of the dichroic signal at the Dy $\mathrm{M}_{5}$ and $\mathrm{Ti} \mathrm{L}_{3}$ edges using X-ray Resonant Magnetic Scattering (XRMS) to obtain elementselective magnetic hysteresis cycles as function of temperature, see Figure 4. Both Ti and Dy show a hysteresis cycle at low temperature (Fig. 4a and 4c), with a magnetic transition occurring around $60 \mathrm{~K}$, in good agreement with the SQUID data for thick samples. This observation supports the globally ferrimagnetic magnetic order of $\mathrm{DyTiO}_{3}$ observed in Refs. ${ }^{[12,13]}$ (see bottom sketch in Fig. 4b). The main point is that while the titanium develops a purely ferromagnetic response, the dysprosium shows a superposition of a ferromagnetic and a paramagnetic response. The signal at the Dy edge is thus the superposition of the response of Dy ions coupled with the $\mathrm{Ti}^{3+}$ by exchange and of uncoupled (paramagnetic) Dy ions in a $\mathrm{Ti}^{4+}$ environment. This demonstrates the causality between the change in the titanium valence at the sample surface and the living dead layer phenomena. We can now come back to the analysis of Fig. $2 \mathrm{~b}$ and $2 \mathrm{~d}$, assuming that the paramagnetic signal with a large magnetization at high field 


\section{WILEY-VCH}

comes from uncoupled $\mathrm{Dy}^{3+}$ ions (model 2). The moment of such a $4 \mathrm{f}^{10}$ system is $10.6 \mu_{\mathrm{B}}$ per ion at saturation. From simulations of its Brillouin function

$$
m(H)=g J\left(\frac{2 J+1}{2 J} \operatorname{coth}\left(\frac{2 J+1}{2 J} x\right)-\frac{1}{2 J} \operatorname{coth}\left(\frac{1}{2 J} x\right)\right)
$$

where $\mathrm{g}$ is the Landé factor, $x=\frac{g \mu_{0} \mu_{B} J H}{k_{B} T}$ and $J=\frac{15}{2}$, we determine that the moment is $8.3 \mu_{\mathrm{B}}$ at our maximum field of $5 \mathrm{~T}$. We thus set $m_{P}=8.3 \mu_{\mathrm{B}} / \mathrm{f}$.u. The fits of $m_{\text {total }}$ vs $t$ for both thin film series are shown as blue lines. The agreement with the data is good within error bars and yields $t_{P}=4-5 \mathrm{~nm}$ for the pure Ar series and $t_{p}=1-2 \mathrm{~nm}$ for the $\mathrm{O}_{2}$ series. To sum up, both the XAS and SQUID data point to the presence of a 4-5 nm thick dead layer at the surface of the films, comprising $\mathrm{Ti}^{4+}$ ions and behaving as a paramagnet (see top sketch of Fig. 4b). The effective dead layer inferred from (in situ) XPS is thinner, $1.3 \mathrm{~nm}$, which probably reflects some additional surface overoxidation after air exposure.

Finally, we have explored several possibilities to "revive" the magnetic dead-layer. Following $\mathrm{Xu}$ et $\mathrm{al}^{[7]}$ the presence of the titanium $4+$ at the surface of rare-earth titanates might be explained by oxygen ions migrating to interstitial sites during the cooling process. We have thus attempted to reduce the surface of a $\mathrm{DyTiO}_{3}$ film by capping it with different materials. Starting with a $10 \mathrm{~nm}$ DTO film with a high $\mathrm{Ti}^{4+}$ level and relatively poor magnetic response, we first investigated the effect of a 5 unit-cell cap of $\mathrm{LaAlO}_{3}$ grown by PLD in the same conditions as the DTO film. In Figure 5a we compare the XPS response of the DTO film right after growth, and of the very same film after in situ capping. We see that the $3+$ components of the titanium $2 p$ levels are enhanced by the addition of 5 u.c. of LAO; we estimate that the $3+/ 4+$ ratio increases from 29:71 to 45:55. To see a clear effect on the magnetic properties, we grew a similar sample with a $30 \mathrm{~nm}$ LAO capping layer, and also studied films with $30 \mathrm{~nm} \mathrm{Au}$ and Al caps. The Figure 5b shows a clear improvement of the ferrimagnetic properties of the DTO capped with the reducing materials ( $\mathrm{LAO}$ and $\mathrm{Al}$ ), with a much higher remanent magnetization, 


\section{WILEY-VCH}

and a lower magnetization at $5 \mathrm{~T}$. Both $\mathrm{Al}$ and $\mathrm{LAO}$ are reducing materials and it is thus expected that the excess of oxygen at the interface with DTO will be partially diminished. On the contrary Au, a noble metal, does not have such a strong effect. These results suggest that a reducing process takes place to restore the $3+$ valence at the surface by taking the excess oxygen out.

In summary, we have shown the presence of a 4-5 nm thick "living-dead" layer at the surface of a ferrimagnetic rare earth titanate, $\mathrm{DyTiO}_{3}$. In this region, the valence of the Ti strongly deviates from $3+$ towards $4+$, which empties the Ti $3 d$ band thus killing the ferrimagnetic response but exhuming a massive paramagnetic signal from uncoupled $\mathrm{Dy}^{3+}$ ions. Through in situ XPS and ex situ XAS at the Ti edge with different probing depth, we have found that while in the bulk of the films $\mathrm{Ti}$ is clearly $3+, \mathrm{Ti}^{4+}$ is progressively more visible as the most superficial regions are probed. The observed concomitant decrease of the remanent magnetization and increase of the saturation magnetization when reducing film thickness indicates the presence of a paramagnetic dead layer. This picture of magnetically coupled Ti and Dy ions coexisting with some paramagnetic Dy is corroborated by element specific hysteresis cycles. Our results shed light on the reported anomalies in XAS and XMCD spectra in nominally trivalent Ti oxides, and suggest severe materials compatibility constrains for titanate-based quantum heterostructures. 


\section{WILEY-VCH}

\section{Experimental section}

\section{Sample preparation}

The samples have been grown on (001)-oriented LAO substrates (from CrysTec GmbH). A polycrystalline target (PiKem) consisting of a mixture of $\mathrm{Dy}_{2} \mathrm{O}_{3}, \mathrm{TiO}_{2}$ and $\mathrm{Dy}_{2} \mathrm{Ti}_{2} \mathrm{O}_{7}$ with an overall 1:1 Dy:Ti ratio was ablated by a Coherent Compact KrF (248 nm) excimer laser at a repetition rate of $2 \mathrm{~Hz}$ and with a fluence of $2 \mathrm{~J} / \mathrm{cm}^{2}$. The deposition has been done at a substrate temperature of $900{ }^{\circ} \mathrm{C}$. Two different atmospheres are suitable for the growth of DTO, $4 \times 10^{-7}$ mbar of $\mathrm{O}_{2}$ or $1 \times 10^{-3}$ mbar of pure Ar. The Ar has been used to control the scattering of lighter species (mainly $\mathrm{O}^{-}$) and the pressure of $1 \times 10^{-3}$ mbar is optimum to that effect ${ }^{[29,30]}$. The substrate-to-target distance was set to $4.5 \mathrm{~cm}$. After deposition the samples were cooled in the same growth atmosphere at $50{ }^{\circ} \mathrm{C} \cdot \mathrm{min}^{-1}$. The LAO capping was grown in the same condition as the DTO deposition. A PLASSYS magnetron sputtering at room temperature has been used to grow the $30 \mathrm{~nm}$ layers of $\mathrm{Au}$ and $\mathrm{Al}$.

\section{Structural characterization}

We used an Panalytical Empyrean X-rays diffractometer to acquire the $2 \theta$ - $\omega$ scans. For that we used for the incident beam a $\mathrm{Ge}(220)$ mirror prefix module with a $1 / 8^{\circ}$ receiving slit and a mask of $2 \mathrm{~mm}$. For the detector optic a $1 / 4^{\circ}$ anti-scatter slit was placed before a 3D PIXcel detector. The absence of Laue fringes in the $2 \theta-\omega$ scans may be ascribed to the surface roughness of about $1 \mathrm{~nm}$ and to the composition gradient near the surface. Film thickness was determined through X-ray reflectometry on the same setup.

\section{$X$-ray photoemission spectroscopy}

X-ray photoemission spectroscopy was performed using an $\mathrm{Mg} \mathrm{K}_{\alpha}$ source ( $\left.\mathrm{h} v=1253.6 \mathrm{eV}\right)$. Spectra analysis was carried out with the CasaXPS software. The depth detection limit has been previously measured to be 15 u.c. of LAO $(\approx 5.6 \mathrm{~nm})^{[31]}$. 


\section{WILEY-VCH}

$X$-ray resonant magnetic scattering

The XRMS measurements were carried out at the synchrotron radiation source BESSY II of the Helmholtz-Zentrum Berlin at the VEKMAG end station installed at the PM2 beamline ${ }^{[32]}$. Element selective magnetic hysteresis loops were obtained in reflection geometry at energies across the Dy $M_{5^{-}}$and Ti $L_{3}$ edges showing maximum dichroic signal at a gracing incidence angle of $15^{\circ}$ for the the incoming circularly polarized radiation. After fixing the energy at one of the maximum of dichroism in reflection mode at a $15^{\circ}$ angle, we plotted this value as a function of field to obtain the hysteresis cycle of Figure 4.

$X$-ray absorption spectrocopy

The XAS experiments was performed at the Swiss Light Source (SLS) on the XTreme beamline $^{[33]}$. We obtain the XAS spectra under a $5 \mathrm{~T}$ magnetic field at $20 \mathrm{~K}$. They have been acquired in three detection modes Total Electron Yield (TEY), Total Fluorescence Yield (TFY), X-rays Excited Optical Luminescence (XEOL). The TEY was acquired by measuring the ground current and the fluorescence data in TFY (without energy selection; however, the photodiode for TFY is capped with an Al foil which ensures that visible photons and electrons do not reach the diode). For XEOL measurements, a similar type of photodiode was used, sensitive to both visible photons and X-rays but mounted at the back of the sample ensuring that no X-rays are transmitted to the photodiode.

\section{Acknowledgements}

The authors acknowledge fruitful discussions with N. Reyren, D.C. Vaz, A. Sander and A. Barthélémy. This work received support from the European Research Council Consolidator Grant \#615759 “MINT,” the région Île-de-France DIM “Oxymore” (project "NEIMO”), and the ANR project "NOMILOPS." R.A. thanks the French Ministry of Higher Education and 


\section{WILEY-VCH}

Research and CNRS for financing his PhD thesis. J.S. thanks CNRS and the Scholarship program Alembert funded by the IDEX Paris-Saclay (ANR-11-IDEX-0003-02) for financing his stay at CNRS/Thales. We thank HZB for the allocation of synchrotron radiation beamtime. The research leading to these results has received funding from the European Community's Seventh Framework Programme (FP7/2007-2013) under grant agreement n. ${ }^{\circ} 312284$.

Received: ((will be filled in by the editorial staff))

Revised: ((will be filled in by the editorial staff)) Published online: ((will be filled in by the editorial staff)) 


\section{WILEY-VCH}

\section{References}

[1] H. D. Zhou, J. B. Goodenough, J. Phys. Condens. Matter 2005, 17, 7395.

[2] M. Mochizuki, M. Imada, New J. Phys. 2004, 6, 1.

[3] J. E. Greedan, J. Less-Common Met. 1985, 111, 335.

[4] J. Varignon, M. N. Grisolia, D. Preziosi, P. Ghosez, M. Bibes, Phys. Rev. B 2017, 96, 235106.

[5] P. Moetakef, T. A. Cain, D. G. Ouellette, J. Y. Zhang, D. O. Klenov, A. Janotti, C. G. Van De Walle, S. Rajan, S. J. Allen, S. Stemmer, O. Dmitri, A. Janotti, C. G. Van De Walle, S. Rajan, S. James, Appl. Phys. Lett. 2011, 99, 232116.

[6] M. N. Grisolia, J. Varignon, G. Sanchez-Santolino, A. Arora, S. Valencia, M. Varela, R. Abrudan, E. Weschke, E. Schierle, J. E. Rault, J.-P. Rueff, A. Barthélémy, J. Santamaria, M. Bibes, Nature Phys. 2016, 12, 484.

[7] P. Xu, Y. Ayino, C. Cheng, V. S. Pribiag, R. B. Comes, P. V. Sushko, S. A. Chambers, B. Jalan, Phys. Rev. Lett. 2016, 117, 106803.

[8] P. Moetakef, D. G. Ouellette, J. Y. Zhang, T. A. Cain, S. J. Allen, S. Stemmer, J. Cryst. Growth 2012, 355, 166.

[9] M. N. Grisolia, F. Y. Bruno, D. Sando, H. J. Zhao, E. Jacquet, X. M. Chen, L. Bellaiche, A. Barthélémy, M. Bibes, Appl. Phys. Lett. 2014, 105, 172402.

[10] S. C. Chae, Y. J. Chang, S. S. A. Seo, T. W. Noh, D.-W. Kim, C. U. Jung, Appl. Phys. Lett. 2006, 89, 182512.

[11] Y. Cao, P. Shafer, X. Liu, D. Meyers, M. Kareev, S. Middey, J. W. Freeland, E. Arenholz, J. Chakhalian, Appl. Phys. Lett. 2015, 107, 112401.

[12] C. W. Turner, J. E. Greedan, J. Solid State Chem. 1980, 34, 207.

[13] C. W. Turner, M. F. Collins, J. E. Greedan, J. Magn. Magn. Mater. 1981, 23, 265.

[14] M. Bibes, L. Balcells, S. Valencia, J. Fontcuberta, M. Wojcik, E. Jedryka, S. Nadolski, 


\section{WILEY-VCH}

Phys. Rev. Lett. 2001, 87, 67210.

[15] M. Bibes, L. Balcells, S. Valencia, J. Fontcuberta, E. Jedryka, M. Wojcik, S. Nadolski, Thin Solid 2001, 400, 85.

[16] R. Peng, H. C. Xu, M. Xia, J. F. Zhao, X. Xie, D. F. Xu, B. P. Xie, D. L. Feng, Appl. Phys. Lett. 2014, 104, 81606.

[17] B. Kim, D. Kwon, J. H. Song, Y. Hikita, B. G. Kim, H. Y. Hwang, Solid State Commun. 2010, 150, 598.

[18] Z. Liao, M. Huijben, Z. Zhong, N. Gauquelin, S. Macke, R. J. Green, S. Van Aert, J. Verbeeck, G. Van Tendeloo, K. Held, G. A. Sawatzky, G. Koster, G. Rijnders, Nature Mater. 2016, 15, 425.

[19] M. C. Biesinger, L. W. M. Lau, A. R. Gerson, R. S. C. Smart, Appl. Surf. Sci. 2010, $257,887$.

[20] M. C. Biesinger, B. P. Payne, B. R. Hart, A. P. Grosvenor, N. S. McIntryre, L. W. Lau, R. S. Smart, J. Phys. Conf. Ser. 2008, 100, 12025.

[21] P. Scheiderer, M. Schmitt, J. Gabel, M. Zapf, M. Stübinger, P. Schütz, L. Dudy, C. Schlueter, T.-L. Lee, M. Sing, R. Claessen, Unpublished

[22] S. Tanuma, T. Shiratori, T. Kimura, K. Goto, S. Ichimura, C. J. Powell, Surf. Interface Anal. 2005, 37, 833.

[23] E. Lesne, N. Reyren, D. Doennig, R. Mattana, H. Jaffrès, V. Cros, F. Petroff, F. Choueikani, P. Ohresser, R. Pentcheva, A. Barthélémy, M. Bibes, Nature Commun. 2014, 5, 4291 .

[24] M. Abbate, F. M. F. de Groot, J. C. Fuggle, A. Fujimori, Y. Tokura, Y. Fujishima, O. Strebel, M. Domke, G. Kaindl, J. van Elp, B. T. Thole, G. A. Sawatzky, M. Sacchi, N. Tsuda, Phys. Rev. B 1991, 44, 5419.

[25] M. Haverkort, Z. Hu, A. Tanaka, G. Ghiringhelli, H. Roth, M. Cwik, T. Lorenz, C. Schüssler-Langeheine, S. Streltsov, A. Mylnikova, V. Anisimov, C. de Nadai, N. 


\section{WILEY-VCH}

Brookes, H. Hsieh, H.-J. Lin, C. Chen, T. Mizokawa, Y. Taguchi, Y. Tokura, D.

Khomskii, L. Tjeng, Phys. Rev. Lett. 2005, 94, 56401.

[26] F. Iga, M. Tsubota, M. Sawada, H. B. Huang, S. Kura, M. Takemura, K. Yaji, M.

Nagira, A. Kimura, T. Jo, T. Takabatake, H. Namatame, M. Taniguchi, Phys. Rev.

Lett. 2004, 93, 257207.

[27] M. Mizumaki, Solid State Ionics 2004, 172, 565.

[28] T. J. Regan, H. Ohldag, C. Stamm, F. Nolting, J. Lüning, J. Stöhr, R. L. White, Phys. Rev. B 2001, 64, 214422.

[29] J. Chen, M. Döbeli, D. Stender, M. M. Lee, K. Conder, C. W. Schneider, A. Wokaun, T. Lippert, J. Phys. D. Appl. Phys. 2016, 49, 45201.

[30] J. Chen, M. Döbeli, D. Stender, K. Conder, A. Wokaun, C. W. Schneider, T. Lippert, Appl. Phys. Lett. 2014, 105, 114104.

[31] D. C. Vaz, E. Lesne, A. Sander, H. Naganuma, E. Jacquet, J. Santamaria, A. Barthélémy, M. Bibes, Adv. Mater. 2017, 29, 1700486.

[32] T. Noll, F. Radu, in Proc. MEDSI2016, 2017, pp. 370-373.

[33] C. Piamonteze, U. Flechsig, S. Rusponi, J. Dreiser, J. Heidler, M. Schmidt, R. Wetter, M. Calvi, T. Schmidt, H. Pruchova, J. Krempasky, C. Quitmann, H. Brune, F. Nolting, J. Synchrotron Radiat. 2012, 19, 661. 


\section{WILEY-VCH}
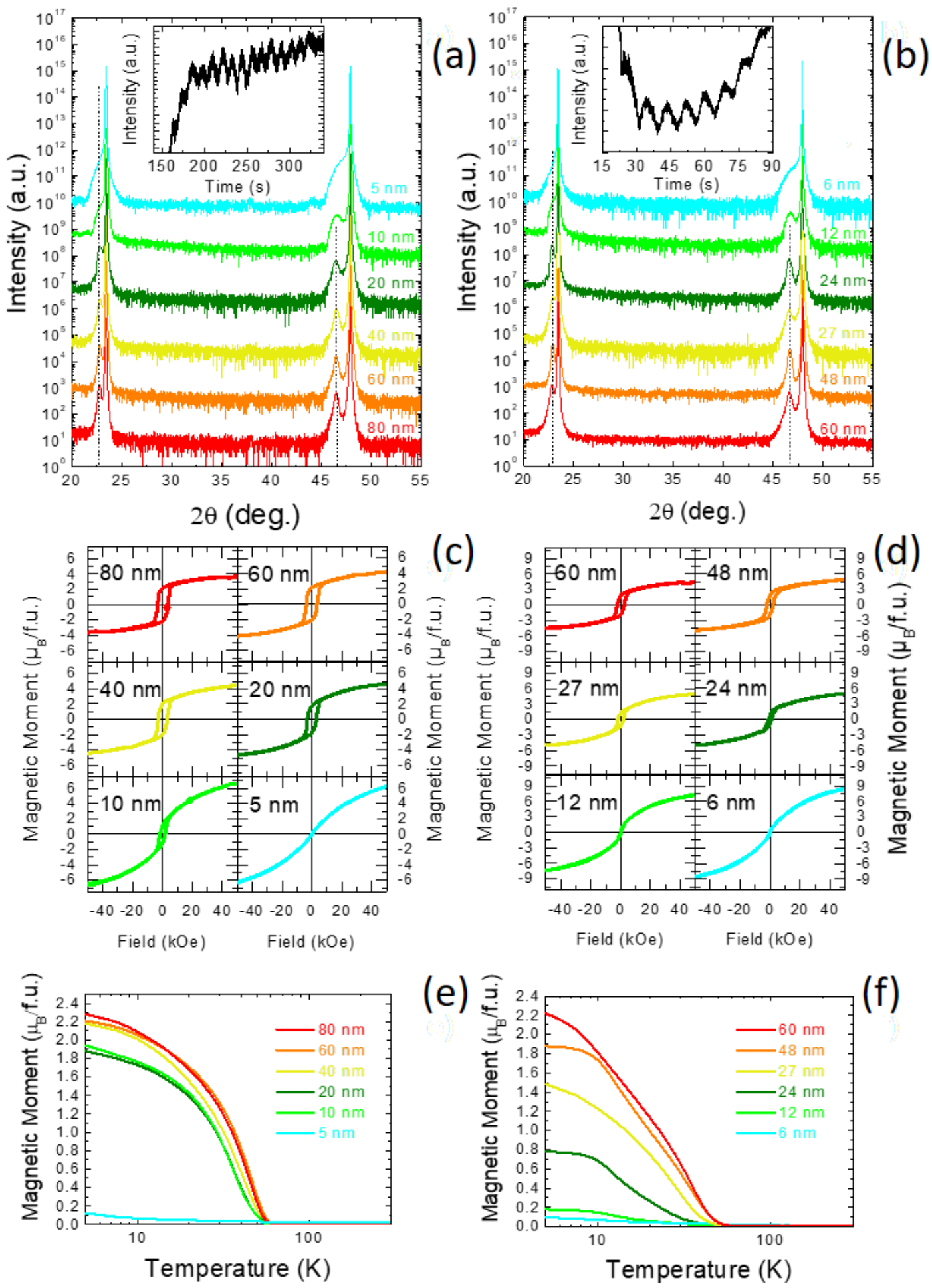

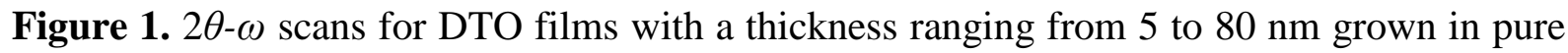
$\operatorname{Ar}(a)$ and low $\mathrm{P}_{\mathrm{O} 2}$ (b). The insets show the dependence of the RHEED intensity during growth. Magnetic hysteresis cycles measured at $4 \mathrm{~K}$ for DTO films grown in pure $\mathrm{Ar}$ (c) and low $\mathrm{P}_{\mathrm{O} 2}$ (d). Temperature dependence of the magnetization at $0.1 \mathrm{~T}$ for DTO films grown in pure $\mathrm{Ar}(\mathrm{e})$ and low $\mathrm{P}_{\mathrm{O} 2}(\mathrm{~b})$. 


\section{WILEY-VCH}
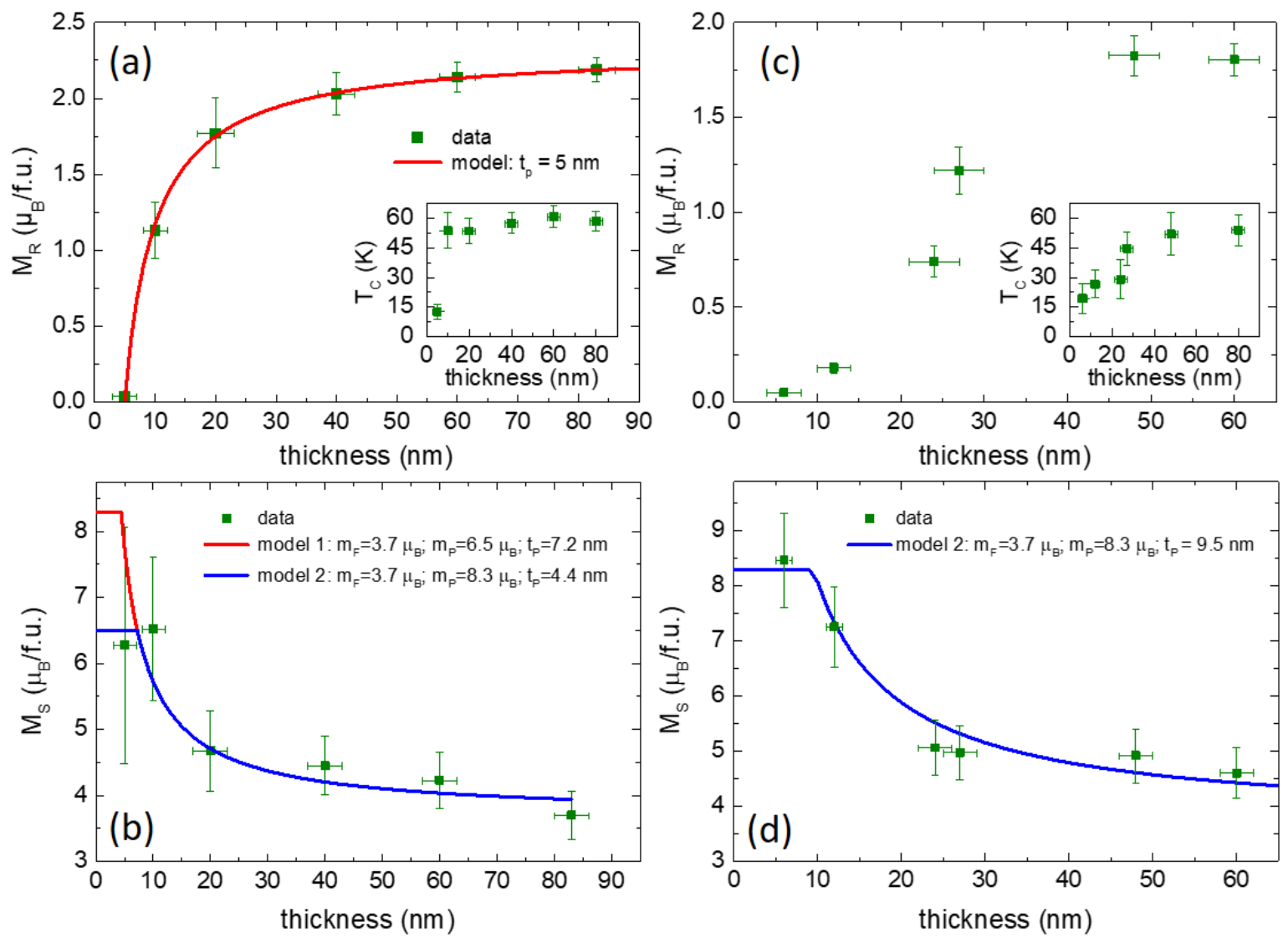

Figure 2. Thickness dependence of the remanent ((a) and (c)) and saturation magnetization ((b) and (d)) for films grown in pure $\operatorname{Ar}((a)$ and (b)) and in oxygen ((c) and (d)). The flat parts at low thickness in the lines in (b) and (d) corresponds to the situation where the sample only consists of a living-dead layer. 


\section{WILEY-VCH}

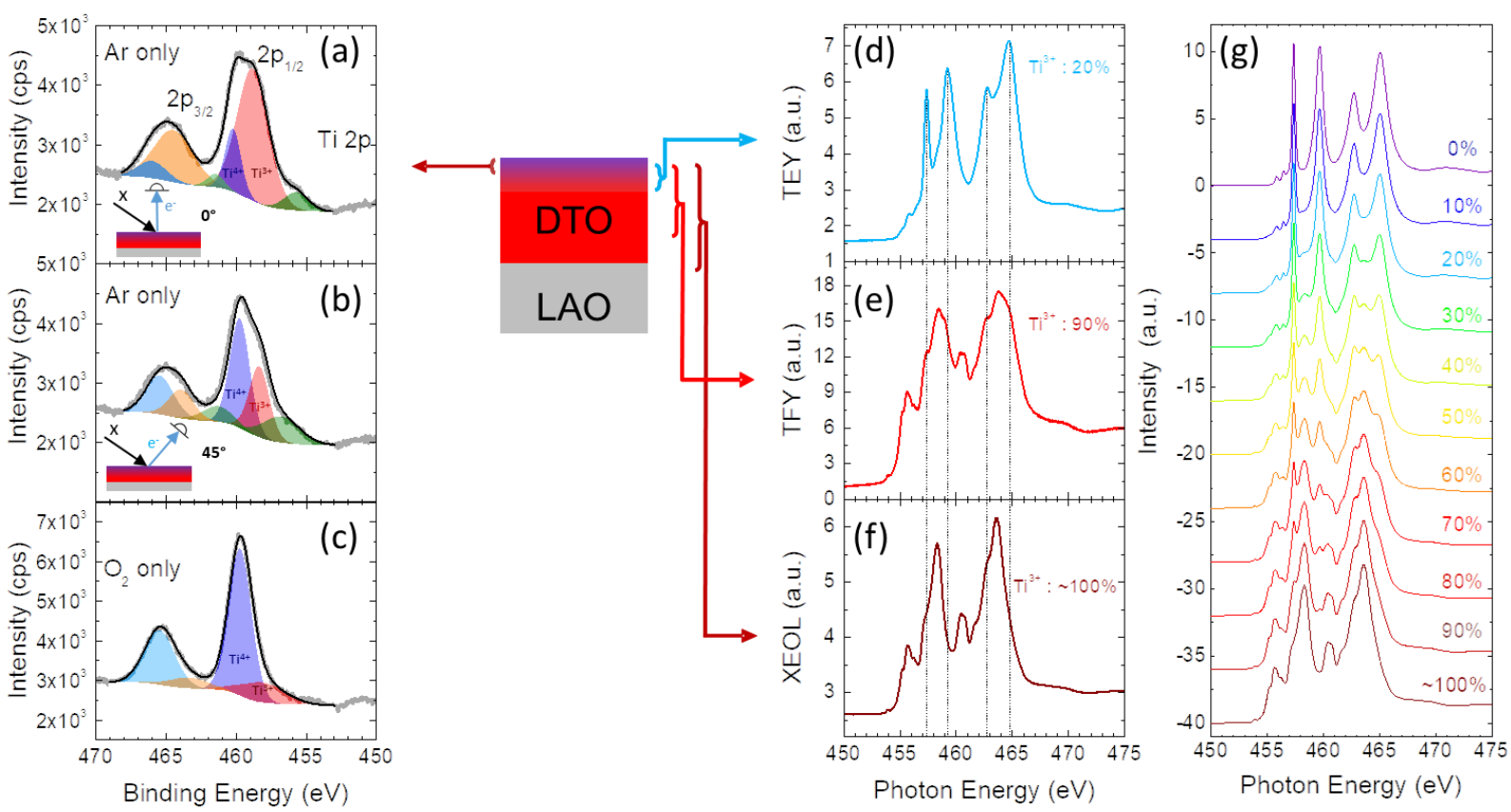

Figure 3. (a-c) XPS spectra of the Ti 2p core levels in $20 \mathrm{~nm}$ DTO films grown in $\operatorname{Ar}((\mathrm{a})$ and (b)) and in oxygen (c), at normal emission ((a) and (c)) and a 45 degrees emission (b). XAS at the Ti L3,2 edge at room temperature in a $20 \mathrm{~nm}$ DTO film grown in pure Ar, collected in TEY mode (d), FY mode (e) and XEOL mode (f). The dotted lines guide the eye to the position of the four main peaks present in (d). (g) Linear combinations of XAS spectra between a reference $\mathrm{Ti}^{4+}$ spectrum and a reference $\mathrm{Ti}^{3+}$ spectrum (see text for details). 


\section{WILEY-VCH}

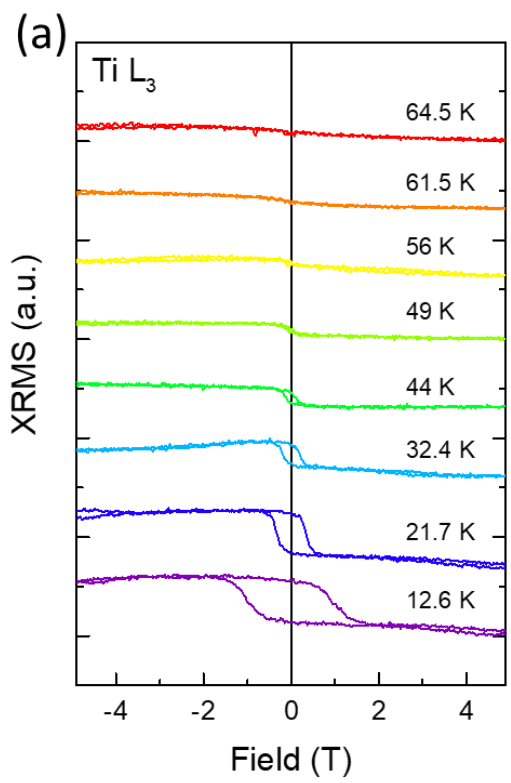

(b)

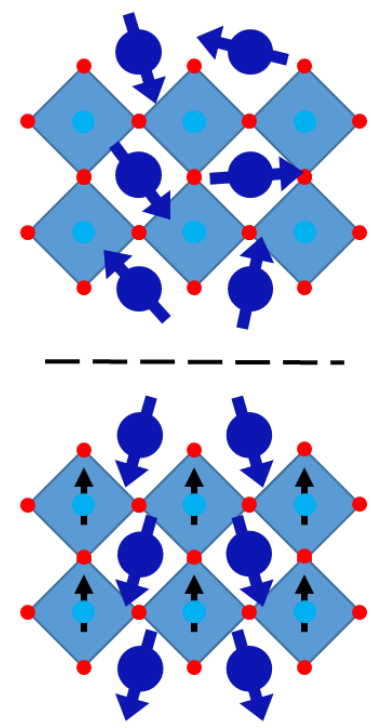

(c)

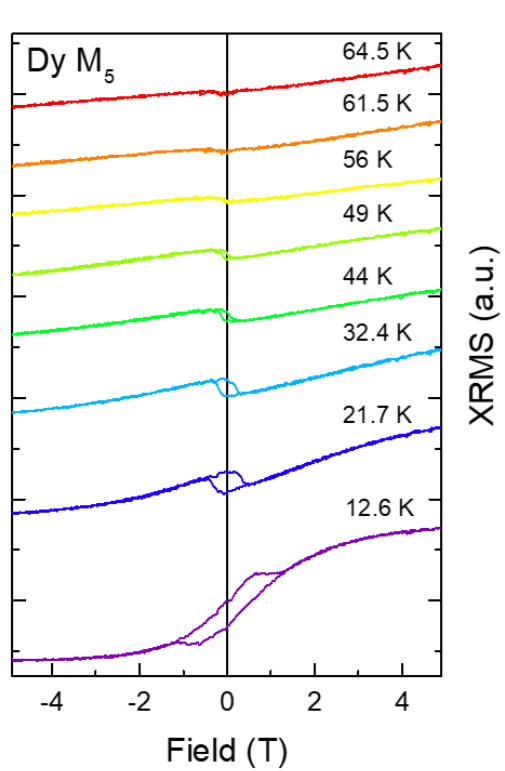

Figure 4. Magnetic field dependence of the XMRS signal at the Ti $L_{3}$ edge (a) and the Dy $M_{5}$ edge (c), at different temperatures, for a $80 \mathrm{~nm}$ DTO film grown in Ar. (b) Sketch of the magnetic order near the surface (top), where Ti is $4+$, carries no magnetic moment and Dy is paramagnetic and deeper in the film (bottom), where Ti is 3+ and the Ti and Dy moments show a canted ferrimagnetic order. 


\section{WILEY-VCH}

(a)

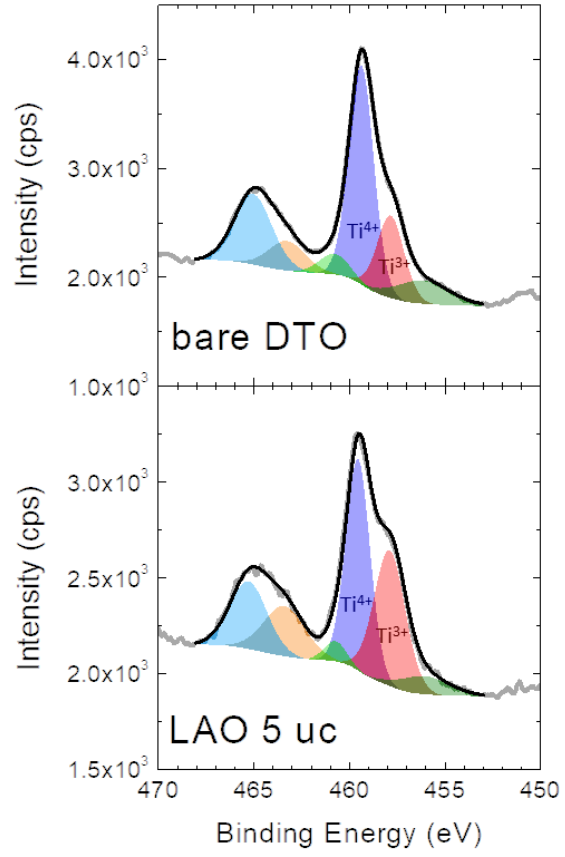

(b)

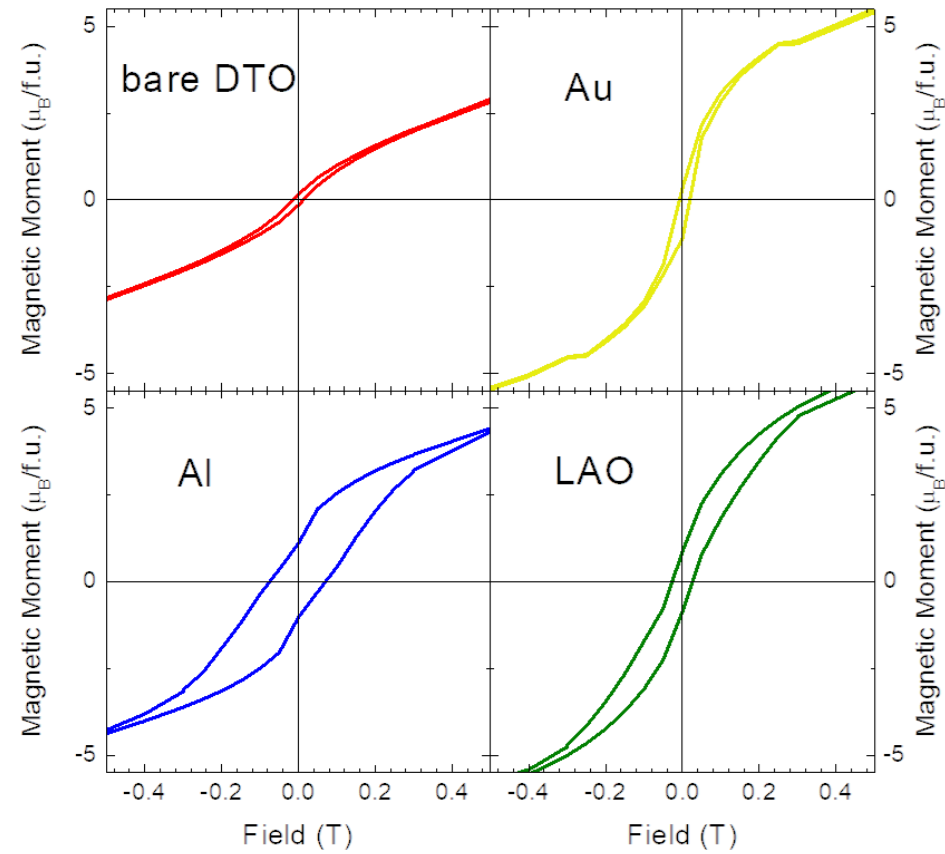

Figure 5. (a) XPS spectra of the Ti $2 p$ core levels for a $10 \mathrm{~nm}$ DTO film, before (top) and after (bottom) capping with 5 unit cells of LAO. (b) Magnetic hysteresis cycles at $4 \mathrm{~K}$ of 10 nm DTO films uncapped, and capped with different materials. 


\section{WILEY-VCH}

The table of contents entry

A peculiar magnetic "dead layer" is detected at the surface of thin films of $\mathrm{DyTiO}_{3}$, a

ferrimagnetic Mott insulator. Depth-dependent X-ray spectroscopy indicate that this layer is associated with a deviation of the Ti valence from 3+ towards 4+ at the film surface, suppressing the magnetic coupling between Ti ions and unleashing a strong paramagnetic response from uncoupled Dy ions. 